\title{
Network Structure and the Emergence of Equity Norm in Nash Bargaining Game
}

\author{
Bo Xianyu ${ }^{1, a}$ \\ ${ }^{1}$ Institue of Logic and Cognition, Sun Yat-Sen University, Guangzhou, China \\ axybmay@sina.cn
}

Keywords: Nash bargaining game; equity norm; social learning; agent based modeling; complex network

\begin{abstract}
Equity norm is an important feature in human society. The present paper studies the effects of different network structures on the emergence of equity norm when agents interact distributed on the complex networks. A revised Nash bargaining game is used to model the emergence of equity norm. This paper focusses whether equity strategy equilibrium can emerge and what effects the network structure plays in the spatial Nash bargaining game. The research results show that equity norm always emerges in the Nash bargaining game no matter what network structure agents are located on. The researches of the present paper reveal that network structure promotes the emergence of the equity regime while different networks have a significant effect on the evolution process.
\end{abstract}

\section{Introduction}

In the past decade, the games on complex network have received many studies in the field of academic research [1]. Many games, such as Prisoner's dilemma game, are studied in the networks which is used as the basic structure of agents' interactions [2-4]. As it is shown in the literature, playing game on the complex networks provides a new way of research. Many difficult issues which is hard to explain such as the cooperation behaviors can be addressed from a new and promising approach now.

However, there are still some important issues deserve more attentions. One of these issues is the equity norm in human society. A norm can be regarded as a pattern of behavior that is a self-enforcing or equilibrium that everyone expect and conform[5]. In game they, there is a tradition which studies the evolution of norms, conventions or rules in human society [5-7]. Young studies the evolution of convention in an n-person population game which is played repeatedly by different agents[5]. Based upon Nash's original idea and Young, Axtell et al.[8] revise it to study the equity norm emergence in a finite population from an agent based approach. With the development of network science, there are some extensions which study the Nash bargaining game in the complex network which are based upon Axtell et al.' s model. Thus the effects of spatial structure of agents' interaction on the evolution of equity norm emergence can be examined. However, the spatial structure of agents interaction is limited on lattice or grid[9]. Recently, Santos et al. [10] extend Axtell's model to study the equity norm emergence on the small world network and the diffusion of equity norm.

Since the equity norm plays an important role in the evolution, maintenance and development of a social system, it is required to further the study of the emergence of equity norms in a society. Given the advanced achieved, this paper develops a Nash bargaining game on complex network where agents play the Nash demand game with their neighboring agents based upon the works of Axtell et al. and Santos et al. The purpose of present paper is to study whether equity norm will emerge among population and what is the effect of network structures on this emergence.

\section{The model}

The model is based upon the Nash bargaining game proposed by Axtell et al. In the one-shot Nash bargaining game, each agent will get its demand if the sum of the two demands is not more than 100 percent of the pie. Otherwise, each agent gets nothing. However, in the simplified Nash bargaining 
game, agent has only three options or strategy to choose from: Low (30 percent of the pie), Medium (50 percent of the pie), and High (70 percent of the pie). The M strategy is the equity strategy here. The payoff matrix of the game is shown in the table 1 :

Table 1. The payoff matrix of Nash bargaining game

$$
\begin{array}{cccc} 
& H & M & L \\
\mathrm{H} & 0,0 & 0,0 & 70,30 \\
\mathrm{M} & 0,0 & 50,50 & 50,30 \\
\mathrm{~L} & 30,70 & 30,50 & 30,30
\end{array}
$$

According to the analysis the classical game theory, it is obvious that there are three pure-strategy Nash equilibria: $(\mathrm{L}, \mathrm{H}),(\mathrm{M}, \mathrm{M})$ and $(\mathrm{H}, \mathrm{L})$. It is also obvious that the equity norm is not bound to appear in the simple one-shot Nash bargaining game.

In the spatial evolutionary Nash bargaining game, there are $\mathrm{N}$ agents located on the vertices of the complex network being studied in this model. In the evolution process, each agent plays the Nash bargaining game with his neighborhood agents. The material payoff each agent gets in each time $t$ is the total payoff it has received from the Nash bargaining play with its neighboring agents. The payoff can be described as:

$$
u_{i}=\sum_{j} x_{i}
$$

Where $j$ is the agent which directly connected with agent $i$ on the network and $x_{i}$ is the payoff this agents get from neighborhood agent $j$ when the Nash bargaining game is played between them.

In the initial status of the game, each agent will be randomly assigned a strategy: $\mathrm{L}, \mathrm{M}$ or $\mathrm{H}$. However, the total number of agents assigned L strategy or M strategy or $\mathrm{H}$ strategy is given by the model designer.

During the evolutionary process of Nash bargaining game, every agent will first compute the payoff it has received from the games played with its neighboring agents. Based upon its own payoff and the payoff of its neighboring agents, each agent will update its strategy in the next step of the evolution. If the neighboring agent chosen receives more payoffs than this agent itself, this agent is more likely to imitate the strategy which this neighboring agent uses currently. The strategy transition rule is shown in the following formulae.

$$
w_{s_{i} \rightarrow s_{j}}=\frac{1}{1+\exp \left[\left(\lambda\left(u_{j}-u_{i}\right)\right]\right.}
$$

Here $\lambda$ indicates the noise in the choice.

In the updating way just described, each agent may update his strategy if imitating neighboring agent's strategy may give him more utilities in Nash Bargaining game. If the evolution process comes to a status where all agents choose $\mathrm{M}$ strategy, it can be said that the system is converged to an equity equilibrium or norm.

\section{Simulation results and analysis}

In the simulation of the evolution process of Nash bargaining game on small-world network, RePast Simphony framework is used to build the multi-agent model. In default, there are 5000 agents total in the system. Agents' network topology is built using standard network building algorithm. The network neighborhood size is to 6 by default. The BA network average degree is 6 also for comparison. The parameter $\lambda$ in transition rule is set to a reasonable value 0.1 . By default, in the initial status, the number of agents using strategy $\mathrm{L}, \mathrm{M}$ and $\mathrm{H}$ is the same.

To make our computer experiment's results more robust, in each parameter combination, each simulation is repeated for 100 times and the average results are used as the basis of the following analysis. 


\section{The emergence of equity norm on networks}

First, the emergence of equity norm is studied using various parameter combinations. We do a lot of computer experiments using different networks and parameter combinations. All these simulations results demonstrate that agents' strategies always converge to equity norm through the distributed interaction among the agents in Nash bargaining game on the networks no matter what the network structure may be. It is a little surprising result and it is quite different from previous research results.

The figures in Figure 1 are from the evolution process in the complex network when different networks are used. These figures show the dynamic process of the convergence of the agents' strategies to equity strategy
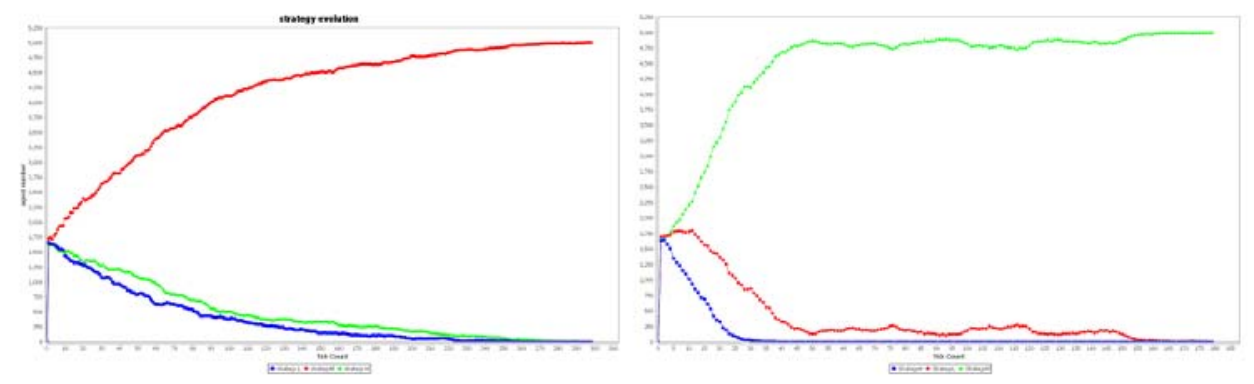

Fig. 1. The convergence of equity on small-world and BA network respectively.

The result about the convergence of agent's strategies to equity equilibrium shows that the equity regime can emerge from the spatial Nash bargaining game. Through learning from their neighboring agents, agents eventually find that equity will bring them more utilities in the long run and the equity preference will emerge in the Nash bargaining game on different networks.

\section{The effect of network structure}

In this paper, this simulation is conducted on small-world and BA network structures given other parameters unchanged at the same time. We let the average neighborhood size be the same for BA network and small-world network first and then various BA networks are used.

The simulation results are shown in the figures:
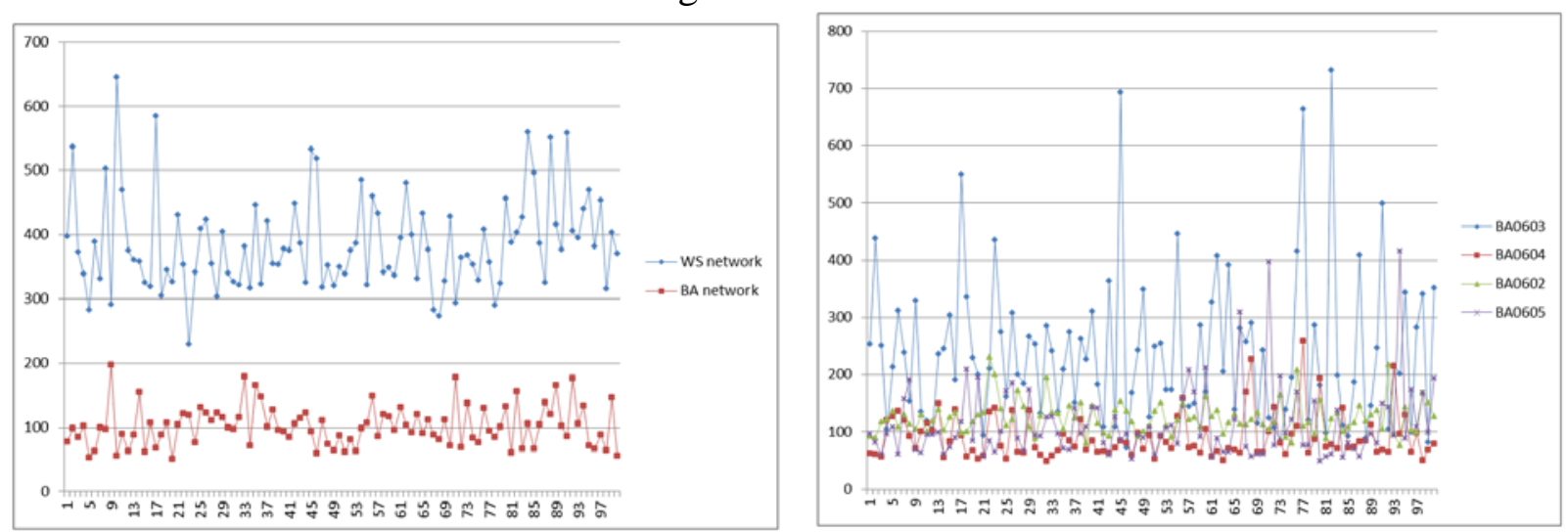

Fig. 2 The covergence time needed on WS small-world network and BA network. The lefe graph is the comparison between small-world network and BA network. The right graph is the result of BA network when BA network initial network size and average network degree are 06 and 04, 06 and 04, 06 and 02, 06 and 05 respectively.

From the simulation results, it can be concluded that the convergence time needed for BA networks are far more less than that of small-world network. The can be explained by the difference of network structures. In BA network, some hub nodes in the network have much more connections 
with other agents; therefore the changes of hub nodes' strategies will affect many agents in the system. While in the small-world network, agents' degree is much more homogeneous. One agent's strategy only affects a small network. Then it is not surprising that agents' strategies will converge quickly to equilibrium than small-world network. Therefore, it is demonstrated that network structure has a great influence on the emergence of equity norm on complex networks.

\section{Conclusion}

Equity norm and its emergence in large society is an important topic for social science and computational science for a long time. In this paper, we have used Nash bargaining game to study the evolution of equity norm in the complex networks. Through computer simulation and analysis, this paper has gotten some interesting results which deserve more attentions. This research results show that networks promote the emergence of equity norm while BA network can speed up the convergence of equity strategy due to the feature of network structures. But there are still some limitations in the present paper. In the present model, three strategies are given first and then agents can imitate their neighboring agents' strategies. What about the situations when agents' strategies are not limited to these three choices? These issues deserve more researches in the coming works.

\section{Acknowledgement}

This work is supported by National Natural Science Foundation of China (Grants No. 71071061, No. 61173019, No. 71231008) and the Major Research Program in Philosophy and Social Science, Ministry of Education (No. 10JZD0006).

\section{References}

[1] György Szabó, Gábor Fáth. Evolutionary games on graphs. Physics Reports [J], 2007(446) 97-216.

[2] György Szabó, Jeromos Vukov, Attila Szolnoki. Phase diagrams for prisoner's dilemma game on two-dimensional lattices. Physical Review E [J], 2005(72) 047107.

[3] Jeromos Vukov, György Szabó, Attila Szolnoki. Evolutionary prisoner's dilemma game on the newman-watts networks. Physical Review E [J], 2008(77) 026109.

[4] Francisco C Santos, Jorge Pacheco. Scale-free networks provide a unifying framework for the emergence of cooperation. Physical Review Letters [J]. 2005(95) 098104.

[5] Peyton Young. The evolution of convention. Econometrica [J], 1993 61(1) 57-84.

[6] Robert Axelrod. An Evolutionary Approach to Norms. American Political Science Review [J], 1986 80(4) 1095-1111.

[7] Peyton Young. Individual strategy and social structure. An evolutionary theory of institutions. Princeton University Press, Princeton, N.J, 1998.

[8] Robert Axelrod: The Emergence of Classes in a Multi-Agent Bargaining Model. Social Dynamics [C], S. N. Durlauf and H. P. Young, Eds., MIT Press, Cambridge, Mass, USA, 2004 191-211,.

[9] David J. Poza, José I. Santos, José M. Galán, Adolfo López-Paredes. Mesoscopic Effects in an Agent-Based Bargaining Model in Regular Lattices. PLoS ONE [J], 2011 6(3) e17661.

[10] José I. Santos, David J. Poza, osé M. Galán, and Adolfo López-Paredes. Evolution of Equity Norms in Small-World Networks. Discrete Dynamics in Nature and Society [J], 2012482481. 